Celgene, Covagen, Crescendo, Janssen, LEO, Lilly, Merck, Novartis, Pfizer, SUN, and UCB; speakers' bureau for AbbVie, Amgen, BMS, Celgene, Genentech, Janssen, Lilly, Pfizer, and UCB, R. Landewé Grant/research support from: Abbott, Amgen, Centocor, Novartis, Pfizer, Roche, Schering-Plough, UCB, Wyeth, Employee of: Director of Rheumatology Consultancy BV, Speakers bureau: Abbott, Amgen, Bristol-Myers Squibb, Centocor, Merck, Pfizer, Roche, Schering-Plough, UCB, Wyeth, S. Mpofu Shareholder of: Novartis, Employee of: Novartis, P. Rahman Consultant for: Abbott, AbbVie, Amgen, BMS, Celgene, Janssen, Novartis, Pfizer and Roche, pharmaceutical companies dealing with biologic agents in Rheumatology, H. Tahir Grant/research support from: Novartis, Pfizer, Consultant for: Abbvie, Novartis, Pfizer, UCB, Eli-Lilly, Janssen, A. Singhal Grant/research support from: AbbVie, Gilead, Sanofi, Regeneron, Amgen, Roche, BMS, Janssen, Lilly, Novartis, Pfizer, UCB, Astra Zeneca, Medlmmune, FujiFilm, Nichi-lko, Mallinckrodt, Speakers bureau: AbbVie, E. Boettcher Consultant for: Amgen, Roche, Eli Lilly, Pfizer, MSD, Novartis, Speakers bureau: Amgen, Roche, Eli Lilly, Pfizer, MSD, Novartis, S. Navarra Consultant for: Pfizer, Novartis, AstraZeneca, Janssen, Astellas, Roche, Speakers bureau: Pfizer, Novartis, AstraZeneca, Janssen, Astellas, Roche, X. Zhu Employee of: Novartis, A. Readie Shareholder of: Novartis stock, Employee of: Novartis, L. Pricop Shareholder of: Novartis stock, Employee of: Novartis, K. Abrams Shareholder of: Novartis stock, Employee of: Novartis

DOI: 10.1136/annrheumdis-2018-eular.6131

\section{OP0307 EFFICACY AND SAFETY OF RISANKIZUMAB, A SELECTIVE IL-23P19 INHIBITOR, IN PATIENTS WITH ACTIVE PSORIATIC ARTHRITIS OVER 24 WEEKS: RESULTS FROM A PHASE 2 TRIAL}

P.J. Mease ${ }^{1 *}$, H. Kellner ${ }^{2}$, A. Morita ${ }^{3}$, A.J. Kivitz ${ }^{4}$, K.A. Papp ${ }^{5}$, S. Aslanyan ${ }^{6}$, B. Berner ${ }^{7}$, S. Chen ${ }^{8}$, A. Eldred ${ }^{8}$, F. Behrens ${ }^{9} .{ }^{1}$ Swedish Med. Ctr. and Univ. of Washington, Seattle, USA; ${ }^{2}$ Private Practice and Div. of Rheumatology KHI Neuwittelsbach, München, Germany; ${ }^{3}$ Dept. of Geriatric and Environmental Dermatology Nagoya City Univ. Graduate Sch. of Med Sci., Nagoya, Japan; ${ }^{4}$ Altoona Ctr. for Clin. Res., Duncansville, USA; ${ }^{5} \mathrm{~K}$ Papp Clin. Res. and Probity Med. Res., Waterloo, Canada; ${ }^{6}$ Boehringer Ingelheim Pharma. Inc., Ridgefield, USA; ${ }^{7}$ Boehringer Ingelheim Pharma GmbH and Co. KG, Biberach, Germany; ${ }^{8}$ Abb Vie Inc., N. Chicago, USA; ${ }^{9} \mathrm{CIRI} /$ Rheumatology and Fraunhofer Institute IME, Translational Med. and Pharmacology, Goethe Univ., Frankfurt, Germany

Background: Interleukin-23 (IL-23), a key regulator of multiple effector cytokines, has been implicated in the pathogenesis of psoriatic lesions, synovitis, enthesitis, and bone erosion. Risankizumab (RZB) is a humanised IgG1 monoclonal antibody that binds to 19 subunit of IL-23, selectively inhibiting this critical cytokine. Objectives: To report the efficacy and safety of different doses of RZB in patients (pts) with active psoriatic arthritis (PsA) over 24 weeks.

Methods: In this double-blind, parallel-design, dose-ranging Phase 2 study, pts with active PsA (stratified by prior TNFi use and concurrent MTX use) were randomised in a 2:2:2:1:2 ratio to receive RZB (150 mg at weeks [Wks] 0, 4, 8, 12, and 16 [Arm 1], $150 \mathrm{mg}$ at Wks 0, 4, and 16 [Arm 2], $150 \mathrm{mg}$ at Wks 0 and 12 [Arm 3], $75 \mathrm{mg}$ single dose at Wk 0 [Arm 4]) or matching placebo (PBO, Arm 5). Pts completing Wk 24 visit had an option to enter a separate open-label extension (OLE) study; pts not entering the OLE were followed until Wk 32. Efficacy assessments included ACR20/50/70, PASI, minimal disease activity (MDA), DAS28(CRP), dactylitis count, SPARCC enthesitis index, pain-VAS, HAQ-DI, and mTSS scores. Results: Of the 185 pts who received the study drug, 173 (93.5\%) completed 16 Wks of treatment and $145(78.4 \%)$ entered OLE at Wk 24. The primary endpoint of ACR20 response at Wk 16 was achieved by pts in each of the RZB arms. ${ }^{1}$ At Wk 24, ACR20/50/70 responses were significantly higher in pts receiving RZB (pooled across all RZB arms) compared with PBO (table 1). PASI75/90/100 responses at Wk 24 were significantly higher in RZB-treated pts compared with PBO. At Wk 24, RZB-treated pts achieved significantly higher MDA responses as well as greater improvements in DAS28(CRP) and Pain-VAS. Improvements in $H A Q-D I$ and enthesitis from $B L$ were numerically greater in RZB arms. At Wk 24, RZB-treated pts (pooled across all RZB arms) showed significant improvement from BL in mTSS compared with PBO. Treatment-emergent adverse events (TEAEs), collected up to Wk 32, were comparable across treatment arms (table 2); the most common TEAE was infection. There were no deaths or cases of tuberculosis in RZB-treated pts; 2 adjudicated major adverse cardiovascular events were reported in RZB arms.
Abstract OP0307 - Table 1. Summary of efficacy results at week $24^{*}$

\begin{tabular}{|c|c|c|c|c|c|c|}
\hline \multirow[b]{2}{*}{ Endpoints } & \multicolumn{5}{|c|}{ Risankizumab (RZB) } & \multirow{2}{*}{$\begin{array}{c}\text { Placebo } \\
\text { Arm } 5 \\
\mathrm{~N}=42\end{array}$} \\
\hline & $\begin{array}{l}\text { Arm } 1 \\
\mathrm{~N}=42\end{array}$ & $\begin{array}{c}\text { Arm } 2 \\
\mathrm{~N}=42\end{array}$ & $\begin{array}{c}\text { Arm } 3 \\
N=39\end{array}$ & $\begin{array}{l}\text { Arm } 4 \\
N=20\end{array}$ & $\begin{array}{c}\text { Arms } 1-4 \\
\mathrm{~N}=143\end{array}$ & \\
\hline ACR20 (\%) & 42.9 & 47.6 & $59.0^{* \prime}$ & 40.0 & $48.3^{\circ}$ & 31.0 \\
\hline ACR50 (\%) & 19.0 & & 33.3* & 20.0 & $22.4^{*}$ & 7.1 \\
\hline CR70 (\%) & 11.9 & 11.9 & $15.4^{\circ}$ & 15.0 & 13.3* & 2.4 \\
\hline $\operatorname{PASI} 75(\%)^{\circ}$ & $68.8 \cdots$ & $70.0^{\cdots} \cdots$ & $69.6^{\cdots} \cdots$ & $55.6^{\circ}$ & $67.6^{\cdots} \cdots$ & 14.3 \\
\hline PASI $90(\%)^{\circ}$ & $60.0^{\cdots}$ & $52.9 *$ & $47.6^{*}$ & $55.6^{*}$ & $53.2 \cdots$ & 10.0 \\
\hline PASI $100(\%)^{\circ}$ & $46.7^{*}$ & 35.3" & $28.6^{\circ}$ & $44.4^{\circ}$ & $37.1 \cdots$ & 5.0 \\
\hline IDA (\%) & 19.0 & $28.6^{* *}$ & $25.6^{\circ}$ & $30.0^{\circ}$ & $25.2 \cdots$ & 7.1 \\
\hline DAS28(CRP) ${ }^{c}$ & -1.1 & $-1.4 *$ & $-1.6 \cdots$ & $-1.7 \cdots$ & $-1.4 \cdots$ & -0.6 \\
\hline Dactyitis Count ${ }^{\text {s }}$ & -1.3 & -2.3 & -3.6 & -3.5 & -2.5 & .2 .6 \\
\hline SPARCC Enthesitis Index & -1.3 & $-2.7^{\circ}$ & -1.6 & $-3.4^{\circ}$ & -2.0 & -1.1 \\
\hline Pain-VAS & -6.9 & .11 .2 & $-19.1 *$ & -6.4 & $.11 .5^{\circ}$ & .3 .2 \\
\hline IHAQ-DI & -0.19 & -0.11 & -0.21 & -0.13 & -0.16 & -0.09 \\
\hline nTSS & -0.3 & 0.2 & $-0.5^{\circ}$ & -0.2 & $-0.2^{*}$ & 0.6 \\
\hline \multicolumn{7}{|c|}{ 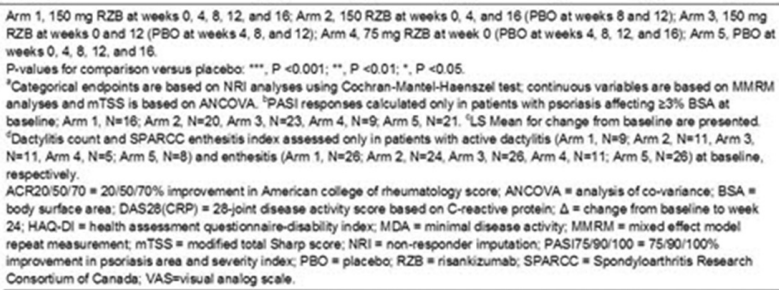 } \\
\hline
\end{tabular}

Abstract OP0307 - Table 2. Overview of treatment-emergent adverse events (TEAEs) over 32 weeks

\begin{tabular}{|c|c|c|c|c|c|}
\hline \multirow[b]{2}{*}{ TEAEs, $n$ (\%) } & \multicolumn{4}{|c|}{ Risankizumab (RZB) } & \multirow{2}{*}{$\begin{array}{c}\text { Placebo } \\
\text { Arm } 5 \\
\mathrm{~N}=42\end{array}$} \\
\hline & $\begin{array}{l}\text { Arm } 1 \\
\mathrm{~N}=42\end{array}$ & $\begin{array}{l}\text { Arm } 2 \\
\mathrm{~N}=42\end{array}$ & $\begin{array}{l}\text { Arm } 3 \\
N=39\end{array}$ & $\begin{array}{l}\text { Arm } 4 \\
N=20\end{array}$ & \\
\hline Any AE & $27(64.3)$ & $22(52.4)$ & $27(69.2)$ & $13(65.0)$ & $31(73.8)$ \\
\hline Drug related AEs" & $8(19.0)$ & $7(16.7)$ & $8(20.5)$ & $4(20.0)$ & $8(19.0)$ \\
\hline Serious $A E^{B}$ & $3(7.1)$ & 0 & $2(5.1)$ & $3(15.0)$ & $2(4.8)$ \\
\hline Drug related serious $\mathrm{AE}^{\mathrm{a}}$ & $1(2.4)$ & 0 & $2(5.1)$ & $1(5.0)$ & 0 \\
\hline Severe AE & $3(7.1)$ & 0 & $2(5,1)$ & $1(5.0)$ & $4(9.5)$ \\
\hline AE leading to drug discontinuation & $3(7.1)$ & 0 & 0 & $1(5.0)$ & $2(4.8)$ \\
\hline Infection ${ }^{c}$ & $16(38.1)$ & $13(31.0)$ & $16(41.0)$ & $6(30.0)$ & $12(28.6)$ \\
\hline Serious infection & 0 & 0 & 0 & $2(10.0)^{6}$ & 0 \\
\hline Serious hypersensitivity & $1(2.4)^{\circ}$ & 0 & 0 & 0 & 0 \\
\hline Adjudicated MACE & $1(2.4)^{\prime}$ & 0 & 0 & $1(5.0)^{0}$ & 0 \\
\hline Malignancy & 0 & 0 & 0 & $1(5.0)^{n}$ & 0 \\
\hline Depression & 0 & 0 & $2(5.1)$ & 0 & 0 \\
\hline \multicolumn{6}{|c|}{ 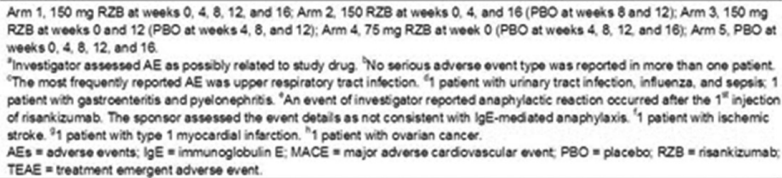 } \\
\hline
\end{tabular}

Conclusions: Pts with active PsA treated with RZB maintained improvement in joint and skin symptoms through 24 wks. RZB-treated pts (pooled across all RZB arms) showed evidence for inhibition of radiographic progression. RZB was welltolerated with no new or unexpected safety findings.

\section{REFERENCE:}

[1] Mease PJ, et al. Arthritis Rheumatol 2017;69(suppl 10).

Acknowledgements: AbbVie and Boehringer Ingelheim (BI) funded the study (NCT02719171); BI contributed to its design and participated in data collection and both participated in data analysis and interpretation of the data, and in writing review, and approval of the publication. AbbVie, $\mathrm{Bl}$, and the authors thank all study investigators for their contributions and the patients who participated in this study. Statistical support: Andrew Topp; medical writing: Deepa Venkitaramani, PhD both of Abbvie.

Disclosure of Interest: P. Mease Grant/research support from: AbbVie, Amgen, Bristol Myers, Celgene, Genentech, Janssen, Leo, Lilly, Merck, Novartis, Pfizer, Sun Pharma, and UCB, Consultant for: AbbVie, Amgen, Bristol Myers, Celgene, Genentech, Janssen, Leo, Lilly, Merck, Novartis, Pfizer, Sun Pharma, and UCB, Speakers bureau: AbbVie, Amgen, Bristol Myers, Celgene, Genentech, Janssen, Leo, Lilly, Merck, Novartis, Pfizer, Sun Pharma, and UCB, H. Kellner Grant research support from: AbbVie, BMS, MSD, Novartis, Pfizer, Roche and UCB, Consultant for: AbbVie, BMS, MSD, Novartis, Pfizer, Roche and UCB, Speakers bureau: AbbVie, BMS, MSD, Novartis, Pfizer, Roche and UCB, A. Morita Grant/ research support from: AbbVie, Eli Lilly Japan K.K., Janssen Pharmaceutical K. K., Kyowa Hakko Kirin Co., Ltd, Leo Pharma, Maruho Co, Ltd, Mitsubishi-Tanabe Pharma, and Novartis, Consultant for: AbbVie, Eli Lilly Japan K.K., Janssen Pharmaceutical K. K., Kyowa Hakko Kirin Co., Ltd, Leo Pharma, Maruho Co, Ltd, Mitsubishi-Tanabe Pharma, and Novartis, Speakers bureau: AbbVie, Eli Lilly Japan K.K., Janssen Pharmaceutical K. K., Kyowa Hakko Kirin Co., Ltd, Leo Pharma, Maruho Co, Ltd, Mitsubishi-Tanabe Pharma, and Novartis, A. Kivitz Grant/ research support from: AbbVie, Boehringer Ingelheim, Celgene, Genentech, Genzyme, Horizon, Janssen, Merck, Novartis, Pfizer, Sanofi, Sun Pharma Advanced 
Research, Regeneron, and UCB, Consultant for: AbbVie, Boehringer Ingelheim, Celgene, Genentech, Genzyme, Horizon, Janssen, Merck, Novartis, Pfizer, Sanofi, Sun Pharma Advanced Research, Regeneron, and UCB, Speakers bureau: AbbVie, Boehringer Ingelheim, Celgene, Genentech, Genzyme, Horizon, Janssen, Merck, Novartis, Pfizer, Sanofi, Sun Pharma Advanced Research, Regeneron, and UCB, K. Papp Grant/research support from: AbbVie, Amgen, Astellas, Baxalta, Baxter, Boehringer Ingelheim, Bristol-Myers Squibb, Celgene, Dermira, Eli Lilly, Forward Pharma, Galderma, Genentech, GlaxoSmithKline, Janssen, Kyowa-Hakko Kirin, Leo Pharma, Medlmmune, Merck-Serono, Merck Sharp and Dohme, Novartis, Pfizer, Regeneron, Roche, Sanofi-Genzyme, Stiefel, Sun Pharma, Takeda, UCB, and Valeant, Consultant for: AbbVie, Amgen, Astellas, Baxalta, Baxter, Boehringer Ingelheim, Bristol-Myers Squibb, Celgene, Dermira, Eli Lilly, Forward Pharma, Galderma, Genentech, GlaxoSmithKline, Janssen, Kyowa-Hakko Kirin, Leo Pharma, Medlmmune, Merck-Serono, Merck Sharp and Dohme, Novartis, Pfizer, Regeneron, Roche, Sanofi-Genzyme, Stiefel, Sun Pharma, Takeda, UCB, and Valeant, Speakers bureau: AbbVie, Amgen, Astellas, Baxalta, Baxter, Boehringer Ingelheim, Bristol-Myers Squibb, Celgene, Dermira, Eli Lilly, Forward Pharma, Galderma, Genentech, GlaxoSmithKline, Janssen, Kyowa-Hakko Kirin, Leo Pharma, Medlmmune, Merck-Serono, Merck Sharp and Dohme, Novartis, Pfizer, Regeneron, Roche, Sanofi-Genzyme, Stiefel, Sun Pharma, Takeda, UCB, and Valeant, S. Aslanyan Employee of: Boehringer Ingelheim, B. Berner Employee of: Boehringer Ingelheim, S. Chen Shareholder of: AbbVie, Employee of: AbbVie, A. Eldred Shareholder of: AbbVie, Employee of: AbbVie, F. Behrens Grant/research support from: AbbVie, Amgen, Celgene, Chugai, Eli Lilly, Genzyme Janssen, Novartis, Pfizer, Roche, Sandoz, and Sanofi, Consultant for: AbbVie, Amgen, Celgene, Chugai, Eli Lilly, Genzyme Janssen, Novartis, Pfizer, Roche, Sandoz, and Sanofi, Speakers bureau: AbbVie, Amgen, Celgene, Chugai, Eli Lilly, Genzyme Janssen, Novartis, Pfizer, Roche, Sandoz, and Sanofi

DOI: 10.1136/annrheumdis-2018-eular.2140

\section{OP0308 EFFICACY AND SAFETY RESULTS OF GUSELKUMAB IN PATIENTS WITH ACTIVE PSORIATIC ARTHRITIS OVER 56 WEEKS FROM A PHASE 2A, RANDOMISED, DOUBLE-BLIND, PLACEBO-CONTROLLED STUDY}

A. Deodhar ${ }^{1}$, A.B. Gottlieb ${ }^{2}$, W.-H. Boehncke ${ }^{3}$, B. Dong ${ }^{4}$, Y. Wang ${ }^{4}$, Y. Zhuang ${ }^{4}$, W. Barchuk ${ }^{5}$, X.L. Xu ${ }^{5}$, E. Hsia ${ }^{4} .{ }^{1}$ Oregon Health and Science University, Portland; ${ }^{2}$ Department of Dermatology, New York Medical College, Valhalla, USA; ${ }^{3}$ Hôpital Universitaire de Genève, Genève, Switzerland; ${ }^{4}$ Janssen Research and Development, LLC, Spring House; ${ }^{5}$ Janssen Research and Development, LLC, San Diego, USA

Objectives: Evaluate efficacy and safety of guselkumab (GUS) in patients (pts) with active psoriatic arthritis (PsA) over 56 weeks (wks).

Methods: Pts w/active PsA (defined as $\geq 3$ tender and $\geq 3$ swollen joints, C-reactive protein $\geq 3 \mathrm{mg} / \mathrm{L}$ ) and $\geq 3 \%$ body surface area (BSA) of plaque psoriasis despite current or previous treatment w/standard-of-care therapies, including previous TNF inhibitor therapy, were eligible to participate and were randomised 2:1 to receive GUS $100 \mathrm{mg}$ subcutaneously or placebo (PBO) at wk 0,4 , and every 8 wks thereafter through wk44. At wk16, pts from either group with $<5 \%$ improvement from baseline in both swollen and tender joint counts were eligible for early escape (EE) to open-label ustekinumab. All remaining PBO pts crossed-over to receive GUS $100 \mathrm{mg}$ at wks24, 28, 36, and 44. At wk56, a posttreatment follow-up visit was conducted. Efficacy post wk24 through wk44 and wk56 was evaluated in pts who did not EE and continued treatment at wk24 (post wk24 efficacy analysis set) based on observed data. The wk24 data in this population were included as a reference.

Results: 149 pts were randomised to receive study agent (PBO: 49, GUS: 100). The study met its primary and all secondary endpoints through wk24. At wk24, 29 pts in the PBO group crossed over to receive GUS, of which 28 completed treatment through wk44. 86 pts in the GUS group continued treatment at wk24 and 84 pts completed treatment through wk44. Post wk24, ACR 20/50/70 and PASI 75/ 90/100 responses improved in PBO to GUS crossover pts and were well-maintained in GUS pts through wk44 (last efficacy assessments while on drug) and wk56 (final follow-up visit) (table 1). The efficacy results from wk24 through wk44 and wk56 are summarised in table 1.

Through wk56, $17.2 \%$ of PBO $\rightarrow$ GUS, $46.0 \%$ of GUS, and $39.5 \%$ of the combined GUS pts had $\geq 1 \mathrm{AEs}$, of which infections and infestations were the most commonly reported $(3.4 \%, 27.0 \%$, and $21.7 \%$, respectively). Post wk24, there was no disproportional increase in overall $A E$ frequency, or infections and infestations among GUS pts with longer exposure. Through wk56, among 129 pts who received GUS, there was 1 pt with malignancy (basal cell carcinoma), 1 pt with 2 serious infections (both pneumonia), 6 pts reported $\geq 1$ SAEs (myocardial infarction, osteoarthritis, pupils unequal, radius fracture, pneumonia, ulcerative keratitis), 2 pts discontinued treatment due to $\mathrm{AEs}, 1 \mathrm{pt}$ had neutropenia meeting $\mathrm{NCl}$ -
CTCAE toxicity grade 3 , and 6 pts were positive for antibodies to GUS. No deaths occurred through wk56.

Abstract OP0308 - Table 1 Efficacy results from Wk24 through Wk44 and Wk56 in post Wk24 efficacy analysis set based on the observed data

\begin{tabular}{|c|c|c|c|c|c|c|}
\hline \multirow[b]{2}{*}{ Efficacy Endpoints } & \multicolumn{3}{|c|}{$\mathrm{PBO} \rightarrow \mathrm{GUS}$} & \multicolumn{3}{|c|}{ GUS } \\
\hline & \begin{tabular}{|l|} 
Week \\
$24^{*}$
\end{tabular} & $\begin{array}{l}\text { Week } \\
44\end{array}$ & $\begin{array}{l}\begin{array}{l}\text { Week } \\
56\end{array} \\
\end{array}$ & $\begin{array}{l}\text { Week } \\
24\end{array}$ & \begin{tabular}{|l} 
Week \\
44
\end{tabular} & \begin{tabular}{|l|} 
Week \\
56
\end{tabular} \\
\hline ACR 20 & $\begin{array}{l}9 / 29 \\
(31.066)\end{array}$ & \begin{tabular}{|l|}
$21 / 28$ \\
$(75.056)$
\end{tabular} & \begin{tabular}{|l|}
$\begin{array}{l}22 / 27 \\
(81.56)\end{array}$ \\
\end{tabular} & \begin{tabular}{|l|}
$57 / 86$ \\
$(66.386)$
\end{tabular} & \begin{tabular}{|l|}
$65 / 84$ \\
$(77.456)$
\end{tabular} & $\begin{array}{l}61 / 83 \\
(73.5 \%)\end{array}$ \\
\hline ACR 50 & \begin{tabular}{|l|}
$5 / 29$ \\
$(17.256)$
\end{tabular} & \begin{tabular}{|l|}
$13 / 28$ \\
$(46.456)$
\end{tabular} & \begin{tabular}{|l|l}
$18 / 27$ \\
$(66.786)$
\end{tabular} & \begin{tabular}{|l}
$34 / 86$ \\
$(39.5 \%)$
\end{tabular} & $\begin{array}{l}39 / 84 \\
(46.465)\end{array}$ & \begin{tabular}{|l|}
$44 / 83$ \\
$(53.056)$
\end{tabular} \\
\hline ACR 70 & \begin{tabular}{|l|}
$1 / 29$ \\
$(3.486)$
\end{tabular} & \begin{tabular}{|l|}
$7 / 28$ \\
$(25.056)$
\end{tabular} & \begin{tabular}{|l|}
$8 / 28$ \\
$(28.656)$
\end{tabular} & \begin{tabular}{|l|}
$14 / 86$ \\
$(16.356)$
\end{tabular} & $\begin{array}{l}22 / 84 \\
(26.256)\end{array}$ & \begin{tabular}{|l|}
$27 / 83$ \\
$(32.5 \%)$
\end{tabular} \\
\hline PASI 75 & $\begin{array}{l}6 / 29 \\
(20.766)\end{array}$ & \begin{tabular}{|l|}
$23 / 28$ \\
$(82.186)$
\end{tabular} & \begin{tabular}{|l|}
$22 / 27$ \\
$(81.556)$
\end{tabular} & \begin{tabular}{|l|}
$71 / 86$ \\
$(82.6 \%)$
\end{tabular} & \begin{tabular}{|l|}
$75 / 83$ \\
$(90.456)$
\end{tabular} & $\begin{array}{l}70 / 82 \\
(85.4 \% 6)\end{array}$ \\
\hline PASI 90 & \begin{tabular}{|l|}
$3 / 29$ \\
$(10.366)$
\end{tabular} & \begin{tabular}{|l|}
$21 / 28$ \\
$(75.056)$
\end{tabular} & $\begin{array}{l}20 / 27 \\
(74.196)\end{array}$ & $\begin{array}{l}61 / 86 \\
(70.996)\end{array}$ & \begin{tabular}{|l|}
$68 / 83$ \\
$(81.966)$
\end{tabular} & \begin{tabular}{|l|}
$64 / 82$ \\
$(78.0 \% 6)$
\end{tabular} \\
\hline PASI 100 & \begin{tabular}{|l|}
$3 / 29$ \\
$(10.366)$
\end{tabular} & \begin{tabular}{|l|}
$19 / 28$ \\
$(67.956)$
\end{tabular} & $\begin{array}{l}15 / 27 \\
(55.656)\end{array}$ & \begin{tabular}{|l|}
$38 / 86$ \\
$(44.25)$
\end{tabular} & \begin{tabular}{|l|}
$53 / 83$ \\
$(63.966)$
\end{tabular} & \begin{tabular}{|l|}
$47 / 82$ \\
$(57.356)$
\end{tabular} \\
\hline Mean (SD) change from baseline in HAQ-Dl score & \begin{tabular}{|l|}
-0.19 \\
$(0.581)$
\end{tabular} & $\begin{array}{l}-0.63 \\
(0.612)\end{array}$ & \begin{tabular}{|l|}
-0.67 \\
$(0.558)$
\end{tabular} & \begin{tabular}{|l|}
-0.46 \\
$(0.530)$
\end{tabular} & \begin{tabular}{|l}
-0.54 \\
$(0.598)$
\end{tabular} & $\begin{array}{l}-0.55 \\
(0.621)\end{array}$ \\
\hline Median (IQR) percent change from base line in Enthesitis Scorea & \begin{tabular}{|l|}
-50.0 \\
$(-100.0$, \\
$0.0)$
\end{tabular} & $\begin{array}{l}-100.0 \\
(-100.0, \\
-60.0)\end{array}$ & \begin{tabular}{|l|}
-100.0 \\
$(-100.0$, \\
$-35.0)$
\end{tabular} & $\begin{array}{l}-100.0 \\
(-100.0, \\
-50.0)\end{array}$ & \begin{tabular}{|l|}
-100.0 \\
$(-100.0$, \\
$-50.0)$
\end{tabular} & $\begin{array}{l}-100.0 \\
(-100.0, \\
-50.0)\end{array}$ \\
\hline Ss of patients with unresolved enthesitisa & \begin{tabular}{|l|}
$12 / 18$ \\
$(66.766)$
\end{tabular} & \begin{tabular}{|l|}
$8 / 17$ \\
$(47.16)$
\end{tabular} & \begin{tabular}{|l|}
$6 / 16$ \\
$(37.596)$
\end{tabular} & \begin{tabular}{|l|}
$26 / 67$ \\
$(38.866)$
\end{tabular} & $\begin{array}{l}25 / 66 \\
(37.996)\end{array}$ & $\begin{array}{l}19 / 65 \\
(29.266)\end{array}$ \\
\hline Median (IQR) percent change from base line in dactylitisb & \begin{tabular}{|l|}
-45.0. \\
$(-70.8$ \\
$0.0)$
\end{tabular} & $\begin{array}{l}-100.0 \\
(-100.0, \\
-100.0)\end{array}$ & $\begin{array}{l}-100.0 \\
(-100.0 \\
-100.0)\end{array}$ & $\begin{array}{l}-100.0 \\
(-100.0, \\
-80.0)\end{array}$ & $\begin{array}{l}-100.0 \\
(-100.0 \\
-100.0)\end{array}$ & $\begin{array}{l}-100.0 \\
(-100.0, \\
-95.0)\end{array}$ \\
\hline \%of patients with unresolved dactylitisb & \begin{tabular}{|l|l|}
$13 / 16$ \\
$(81.3 \% 6)$
\end{tabular} & \begin{tabular}{|l|}
$/ 16$ \\
$(12.5 \%)$
\end{tabular} & \begin{tabular}{|l|}
$1 / 16$ \\
$(6.3 \%)$
\end{tabular} & $\begin{array}{l}20 / 50 \\
(40.086)\end{array}$ & \begin{tabular}{|l|l|l|l|}
$(20.456)$ \\
\end{tabular} & $\begin{array}{l}12 / 48 \\
(25.056)\end{array}$ \\
\hline $\begin{array}{l}\text { Mean (SD) change from baseline in SF-36 physical component } \\
\text { summary (PCS) score }\end{array}$ & $\begin{array}{l}2.13 \\
(7.365)\end{array}$ & $\begin{array}{l}802 \\
(8.647)\end{array}$ & N/A & $\begin{array}{l}7.40 \\
(7.448)\end{array}$ & $\begin{array}{l}8.34 \\
(8.783)\end{array}$ & N/A \\
\hline $\begin{array}{l}\text { Mean (SD) change from baseline in SF-36 mental component } \\
\text { summary (MCS) score }\end{array}$ & $\begin{array}{l}.51 \\
(6.770)\end{array}$ & \begin{tabular}{|l|}
5.53 \\
$(9.013)$
\end{tabular} & N/A & $\begin{array}{ll}5.45 \\
(9.081)\end{array}$ & $\begin{array}{l}4.56 \\
(9.548)\end{array}$ & N/A \\
\hline \%of patients achieving Minimal Disease Activity (MDA) & $\begin{array}{ll}1 / 29 \\
(3.486)\end{array}$ & \begin{tabular}{|l|l|}
$8 / 28$ \\
$(28.6 \%)$
\end{tabular} & N/A & \begin{tabular}{|l|l}
$23 / 86$ \\
$(26.786)$
\end{tabular} & $\begin{array}{l}29 / 84 \\
(34.566)\end{array}$ & $\mathrm{N} / \mathrm{A}$ \\
\hline $\begin{array}{l}\text { "Among the patients with enthesitis at baseline } \\
\text { "Among the patients with dactylitis at baseline } \\
\text { "Measured prior to receiving gusselkumab }\end{array}$ & & & & & & \\
\hline
\end{tabular}

Conclusions: In pts with active PsA and $\geq 3 \%$ BSA of psoriasis, GUS demonstrated substantial benefits on joint symptoms, physical function, psoriasis, enthesitis, dactylitis, and quality of life, and efficacy was well-maintained through wk56. GUS was well-tolerated with no unexpected safety findings in this population after $\sim 1$ year of exposure.

Disclosure of Interest: A. Deodhar Grant/research support from: Janssen Research and Development, LLC, A. Gottlieb Grant/research support from: Janssen Research and Development, LLC, W.-H. Boehncke Grant/research support from: Janssen Research and Development, LLC, B. Dong Shareholder of: Johnson and Johnson, LLC, Employee of: Janssen Research and Development, LLC Y. Wang Shareholder of: Johnson and Johnson, LLC, Employee of: Janssen Research and Development, LLC, Y. Zhuang Shareholder of: Johnson and Johnson, LLC, Employee of: Janssen Research and Development, LLC, W. Barchuk Shareholder of: Johnson and Johnson, LLC, Employee of: Janssen Research and Development, LLC, X. Xu Shareholder of: Johnson and Johnson, LLC, Employee of: Janssen Research and Development, LLC, E. Hsia Shareholder of: Johnson and Johnson, LLC, Employee of: Janssen Research and Development, LLC

DOI: 10.1136/annrheumdis-2018-eular.2059

\section{OP0309 \\ CHARACTERISATION OF CLINICAL BENEFITS IN SUBJECTS CLASSIFIED AS ACR2O NON-RESPONDERS AT WEEK 104 OF APREMILAST TREATMENT: SUBANALYSIS OF 3 LONG-TERM, PHASE III TRIALS}

P. Mease ${ }^{1}$, D. Gladman ${ }^{2}$, A. Kavanaugh ${ }^{3}$, P. Nakasato ${ }^{4}$, B. Guerette ${ }^{4}$, L. Teng ${ }^{4}$, P. Nash ${ }^{5}{ }^{1}$ Swedish Medical Center and University of Washington School of Medicine, Seattle, USA; ${ }^{2}$ Krembil Research Institute, Toronto Western Hospital, Toronto, Canada; ${ }^{3}$ University of California, San Diego; ${ }^{4}$ Celgene Corporation, Summit, USA; ${ }^{5}$ University of Queensland, Brisbane, Australia

Background: The PALACE 1, 2, and 3 trials evaluated the efficacy and safety of apremilast (APR) in subjects with active psoriatic arthritis (PsA) despite prior conventional disease-modifying anti-rheumatic drugs and/or biologics.

Objectives: The aim of this analysis is to further characterise the clinical benefits associated with long-term APR exposure in subjects who failed to achieve an ACR20 response at Week 104.

Methods: Subjects were randomised (1:1:1) to receive placebo (PBO), APR $30 \mathrm{mg}$ BID (APR30), or APR $20 \mathrm{mg} \mathrm{BID}$ at baseline. Subjects who were randomised to APR30 at baseline and classified as ACR20 non-responders (ACR20NRs) at Week 104 were considered for this analysis. At Weeks 24, 52, and 104, ACR core components were examined as well as the proportions of 